\title{
Non-functioning pituitary adenoma underwent surgery: A multicenter retrospective study over the last four decades (1977- 2015)
}

\author{
Pedro Iglesias ${ }^{a}$, Karina Arcano ${ }^{\mathrm{a}}$, Vanessa Triviño ${ }^{\mathrm{b}}$, Paula García-Sancho ${ }^{\mathrm{c}}$, \\ JuanJoséDíez $^{\mathrm{a}, \mathrm{d}}$, Fernando Cordido ${ }^{\mathrm{b}}$, Carles Villabona ${ }^{\mathrm{c}}$ \\ ${ }^{a}$ Department of Endocrinology, Hospital Universitario Ramón y Cajal, Madrid, Spain \\ ${ }^{b}$ Department of Endocrinology, Complejo Hospitalario Universitario A Coruña, A Coruña, Spain \\ ${ }^{c}$ Department of Endocrinology, Hospital Universitario de Bellvitge, Barcelona, Spain \\ ${ }^{d}$ Departament of Medicine, Universidad de Alcalá de Henares, Madrid, Spain
}

\begin{abstract}
Objective. To assess clinical features, diagnostic procedures, therapies and outcomes in patients with clinically nonfunctioning pituitary adenomas (NFPAs) surgically treated over the last four decades.

Design and methods. A multicenter retrospective study in NFPA patients periodically followed up in specialized neuroendocrinology units who underwent surgery in the period 1977-2015 was performed.

Results. A total of 131 patients were studied [66 women (50.4\%); mean age 52.6 \pm 14.8 years (range, 15-82)]. Median diameter of the adenoma was $2.6 \mathrm{~cm}$ (interquartile range, 2.0-3.1). The most frequently type of surgery used was endoscopic endonasal surgery $(58.5 \%)$ followed by microscopic transsphenoidal surgery (37.4\%). Radiation therapy was used in 19 patients $(14.5 \%)$. Ki-67 labeling index performed in 54 patients was $\leq 2 \%$ in $70 \%$ samples. After a median follow-up time of 57 months ( 25 to 128 months), tumor diameter significantly decreased to $0.9 \mathrm{~cm}$ $(0-1.8 \mathrm{~cm}), p<0.001$. Multivariant analysis showed that endoscopic endonasal surgery (HR 2.74, 1.06-6.87, $p=0.036$ ) and radiotherapy (HR $0.04,0.02-0.65, p=0.024$ ) behaved as positive and negative predictors, respectively, of tumor absence in the follow-up. Endoscopic endonasal surgery (HR 6.71, 1.45-31.05, $p=0.015$ ) was the only positive predictor for complete cure in the follow-up.

Conclusion. NFPAs surgically treated in Spain are usually macroadenomas diagnosed around the sixth decade of life with no sex predilection. Type of surgery is associated with clinical outcome. Endoscopic endonasal surgery behaves as a positive predictor for the absence of tumor imaging and complete cure in the follow up.
\end{abstract}

Keywords

Pituitary adenoma; Non-functioning pituitary adenoma; Surgery; Multicenter study; Gonadotropinoma 


\section{Introduction}

Today, pituitary adenoma (PA) is the third leading cause of intracranial neoplasia, following to meningioma and glioma [1], [2], [3], [4]. According to some surveys, its incidence has almost doubled over a period of 30 years, from 6 to 11 cases per million inhabitants/year [5], with a clinical prevalence around 1 case per 1000 inhabitants in recent studies [6]. The prevalence of PA in imaging studies is around $22.5 \%$, with wide range (1-40\%); while in autopsy studies is $14.4 \%$ (range 1-35\%) [1].

PA can be functioning and nonfunctioning, according to the presence or absence of hormonal hypersecretion. The main types of PA are clinically non-functioning pituitary adenomas (NFPAs), prolactinomas, growth hormone $(\mathrm{GH})$ secreting adenomas or somatotropinomas and corticotropin (ACTH) secreting adenomas or corticotropinomas. The frequency of the different types of PA is different and varies with age. While prolactinomas are the most common PA in young patients (2nd-4th decade of life), from age 40, the most prevalent are NFPAs [7].

NFPAs constitute about a third of all PA and its incidence has been estimated at 1.79/100,000 inhabitants/year [8]. Its prevalence is around 26-90 cases per million and constitute 50\% of pituitary tumors in large surgical series [8], [9]. In clinical series its prevalence is much greater around 400 per million [6].

NFPAs are the pituitary macroadenomas $(\geq 1 \mathrm{~cm})$ more frequent in adults and include primarily gonadotropinomas, which produce, although most of them do not secrete, gonadotropins or their subunits (mainly subunit alpha) followed by null cell adenomas, and finally other silent PA, such as corticotropinomas, somatotropinomas, and prolactinoma. Depending on the size, these tumors may be symptomatic or not. Macro-NFPAs are accompanied by considerable morbidity, usually associated with tumor mass effect causing neuro-ophthalmological symptoms and pituitary hormonal failure. On the contrary, micro-NFPAs $(<1 \mathrm{~cm})$ are usually asymptomatic incidental findings on imaging studies. Treatment of choice is transsphenoidal surgery in symptomatic cases with the use or not of adjuvant radiotherapy in the case of persistent or recurrent disease, and periodic observation in asymptomatic tumors [10], [11], [12], [13], [14].

NFPAs series published to date in our country are scarce and have been usually performed in a small number $(\sim 30-50)$ of patients from a single hospital and with a short follow-up [15], [16], [17]. On the other hand, efficacy and surgical outcomes of the different surgical techniques in these tumors have not been clearly conclusive. In the present study we retrospectively reviewed the clinical characteristics, treatments used, pathological findings and clinical outcome of a large cohort of patients with NFPAs surgically treated in the last four decades in three Spanish tertiary hospitals. The main objectives were to study the relationship between different surgical techniques (microscopic and endoscopic) with absence of tumor and complete cure (absence of tumor and adenohypophysis normofunction) in the last medical visit. 


\section{Patients and methods}

\subsection{Patients}

A retrospective multicenter study of NFPAs underwent surgery between 1977 and 2015 in the Endocrinology department of three Spanish hospitals (Hospital Universitario Ramón y Cajal, Madrid; Complejo Hospitalario Universitario de A Coruña, A Coruña; and Hospital Universitario de Bellvitge, Barcelona) was performed. All registered cases of NFPA in each Endocrinology department during the study period were analyzed and all clinical records of the patients who underwent surgery were reviewed.

Inclusion criteria were the histological demonstration of the tumor and the absence of clinical symptoms compatible with pituitary hyperfunction. For homogeneity, MEN 1 patients $(n=2)$ were excluded from the study. Patient percent distribution according to different hospitals included was: Hospital Ramón y Cajal, Madrid, $n=54$ (41.2\%); Hospital Universitario A Coruña, $n=42$ (32.1\%); and Hospital de Bellvitge, $n=35$ (26.7\%). Median follow-up was 57 months (interquartile range, 25 to 128 months).

\subsection{Methods}

In every patient the following clinical parameters were recorded: age at diagnosis, personal and family history, drugs at the time of diagnosis, association or not with multiple endocrine neoplasia, main complaint at consultation, neuro-ophthalmological and endocrinological signs and symptoms and serum baseline hormonal determinations (PRL, GH, IGF-1, ACTH, cortisol, TSH, free T4, FSH, LH, estradiol in women and testosterone in men). The presence of hypopituitarism (partial or complete), number of axes involved, the presence of diabetes insipidus, and hyperprolactinemia was registered. Imaging study at diagnosis and the degree of visual impairment were also evaluated.

Date of surgery, type and surgery technique, and surgical complications were recorded. Histopathological and immunohistochemical study of NFPAs was investigated. Lastly, after surgery, each patient was assessed on his/her last clinical visit analyzing clinical and laboratory data, the need of medical therapy and/or radiotherapy, tumor persistence/recurrence or clinical cure (absence of pituitary tumor in imaging study and normal pituitary function).

\subsection{Statistical analysis}

For quantitative variables, results are expressed as mean \pm SD for normally distributed data, (age) and as median (interquartile range) for nonparametric data (time of follow-up and maximal tumor diameter). Adjustment to normal distribution was tested by the Kolmogorov test. Categorical variables are described as percentages. For comparisons of means between two groups of patients the Student $t$-test was used for normally distributed data (age), and the Mann-Whitney test to compare the medians of two different groups of patients (comparison of maximal tumor diameter in patients with and without hypopituitarism both before and after surgery). Willcoxon test was used to compare the medians for nonparametric data in the same group of patients (comparison of maximal tumor diameter at diagnosis and at last clinical visit). For categorical comparisons the $\chi^{2}$ test (evaluation of sex, age groups at diagnosis, persistence of tumor at last visit, tumor size group, Ki 67 value group, hyperprolactinemia, complete and partial hypopituitarism, and visual field alteration). Bonferroni correction to the $\alpha$ level was applied when tests with multiple comparisons were performed. Unadjusted and multivariate Cox regression were used to assess the effect of different qualitative variables (sex, age, maximal tumor diameter, suprasellar extension, cavernous sinus invasion, type of surgery (microscopic or endoscopic), and radiotherapy) on the absence of tumor and on magnetic resonance imaging (MRI) and complete cure at the last clinical visit. Hazard ratios (HR) and their $95 \%$ confidence intervals were calculated. Differences were considered significant when $p<0.05$. 


\section{Results}

\subsection{Clinical features}

A group of 131 patients [66 women (50.4\%); mean age $52.6 \pm 14.8$ years (range, 15-82)] was studied. Most patients $(n=69 ; 52.7 \%)$ were diagnosed at the age of 40-60 years. Only 3\% $(n=4)$ of patients had an age lower than or equal to 25 years diagnosis while in $25.9 \%(n=34)$ age at diagnosis was greater than or equal to 65 years. No significant differences in the distribution of patients according to sex and age at diagnosis were found (Fig. 1).

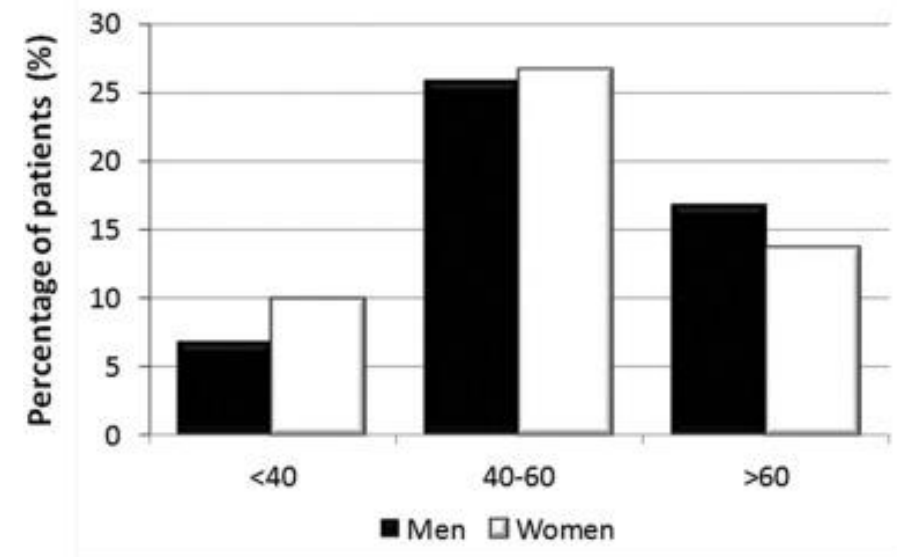

Age at diagnosis (years)

Fig. 1. Percent distribution of 131 patients with NFPA who underwent surgery according to sex and age at diagnosis.

In most patients $(n=103 ; 78.6 \%)$ the tumor was symptomatic, while the rest was an incidental finding in imaging study. The main complaint at initial visit was headache $(n=37)$ that appeared in nearly a third of patients (28.2\%), followed by alterations in the visual field in 17 patients (13\%), mainly as bitemporal hemianopsia, and decreased visual acuity in 12 patients $(9.1 \%)$. Only 2 patients $(1.5 \%)$ were diagnosed after a pituitary apoplexy. After the first clinical evaluation, neuro-ophthalmological symptoms were more frequent than endocrine ones (67.2\% vs. $31.3 \%)$; being visual disturbances and, among them, visual field defects, the main symptoms (55.7\%). Erectile dysfunction, decreased libido (6.9\%), and asthenia $(6.9 \%)$ in men $(8.4 \%)$, and menstrual disorders $(6.8 \%)$ in women, were the most frequent endocrine symptoms.

\subsection{Hormonal study}

Pituitary hormonal function was registered in 104 patients (79.4\%). Of these, 8 patients (7.7\%) had a complete anterior hypopituitarism, while hypopituitarism was partial in 47 patients (45.2\%). Most patients with partial hypopituitarism showed deficiency in one axis $(n=20 ; 19.2 \%)$, followed by 3 -axis deficiency $(\mathrm{n}=14 ; 13.5 \%)$ and 2 -axis deficiency $(\mathrm{n}=13 ; 12.5 \%)$. The most frequently affected hormonal axis was gonadal $(\mathrm{n}=43 ; 41.3 \%)$. 
Hypopituitarism was more common in men than in women $\left(31.7 \%\right.$ vs $21.1 \% ; p=0.005 ; \chi^{2}$ test $)$. Men had a higher prevalence of central hypogonadism $\left(26.9 \%\right.$ vs $14.4 \% ; p=0.002 ; \chi^{2}$ test $)$ and secondary hypothyroidism $(18.3 \%$ vs $9.6 \% ; p=0.02$; test), while women showed a higher prevalence of hyperprolactinemia $\left(25 \%\right.$ vs $9.6 \% ; p=0.003 ; \chi^{2}$ test $)$.

There was a relationship between pituitary function and tumor size at diagnosis. Patients with hypopituitarism showed a tumor size significantly greater than that found in patients without hypopituitarism [2.9 cm $(2.5-3.3 \mathrm{~cm})$ vs $2.2 \mathrm{~cm}(1.9-3.0 \mathrm{~cm}) ; p=0.003$; Mann-Whitney test].

\subsection{Imaging studies}

Most tumors (92.6\%) were diagnosed with MRI and the rest by computed tomography (CT) scan brain. $97.5 \%$ of cases were macroadenomas. 17 of them $(13.1 \%)$ were giant adenomas (diameter $\geq 4 \mathrm{~cm}$ ). $86.4 \%$ of tumors showed suprasellar extension; chiasmal compression occurred in $73.7 \%$ of patients. The invasion of the cavernous sinus was slightly lower (71.6\%). The distribution of tumors according to the Knosp grading scale was as follows: grade $1(8.3 \%)$; grade $2(27.5 \%)$, grade $3(21.1 \%)$, and grade 4 $(14.7 \%)$. In $16.5 \%$ of cases, the invasion of the cavernous sinus was bilateral. The presence of hydrocephalus was unusual $(0.9 \%)$.

Median maximum diameter of the adenoma was $2.6 \mathrm{~cm}$ (interquartile range, 2.0 to $3.1 \mathrm{~cm}$ ). No significant differences in the maximum diameter of adenoma between men and women $[2.8 \mathrm{~cm}(2.2$ to $3.2)$ vs $2.4 \mathrm{~cm}(2.0-3.0)$ were found; ns] neither do among the different age groups analyzed.

\subsection{Treatment and histopathologic results}

Type of surgery was recorded in 123 patients. The most frequently used was endoscopic endonasal surgery $(n=72 ; 58.5 \%)$ followed by microscopic transsphenoidal $(n=46 ; 37.4 \%)$. Transfrontal pituitary surgery was uncommonly performed $(n=5 ; 4.1 \%)$.

The presence of hypopituitarism could be evaluated before and after surgery in 97 patients. Total hypopituitarism was similar after surgery $(8.2 \%$ vs $9.3 \%$, ns) whereas partial hypopituitarism increased significantly $\left(46.4 \%\right.$ vs $53.6 \%, p=0.001 ; \chi^{2}$ test). On the other hand, visual field examination was evaluated before and after surgery in 109 patients. In this case, the percentage of patients with alterations in the visual fields decreased after surgery $(53.2 \%$ vs $19.3 \%, p=0.027$; test) (Table 1$)$.

Table 1. Percent distribution of tumors according to the presence of positivity in immunohistochemical staining for various anterior pituitary hormones $(n=123)$

\begin{tabular}{lll}
\hline Hormone & Number of patients & Percentage $(\%)$ \\
\hline & & \\
LH & 22 & 17.6 \\
FSH & 21 & 16.8 \\
ACTH & 11 & 8.8 \\
GH & 8 & 6.8 \\
PRL & 7 & 5.6 \\
TSH & 4 & 3.2 \\
Negative & 46 & 37.6 \\
& & \\
\hline
\end{tabular}


There was also a relationship between tumor size at diagnosis and pituitary function after surgery. Patients with hypopituitarism after surgery showed a tumor size significantly greater than that found in patients without hypopituitarism [2.8 cm $(2.2-3.3 \mathrm{~cm})$ vs $2.2 \mathrm{~cm}(1.9-3.1 \mathrm{~cm}) ; p=0.021$; Mann-Whitney test].

Nineteen patients $(14.5 \%)$ required a second surgery that was mainly due to persistence/recurrence or worsening of visual disturbances $(n=10,7.6 \%)$. The mean time between the first and second surgeries was $46.8 \pm 47.4$ months (range, $2-156$ months).

The most common surgical complication was transient diabetes insipidus $(n=11 ; 8.4 \%)$, followed by cerebrospinal fluid leak $(n=8 ; 6.1 \%)$, and permanent diabetes insipidus $(n=5 ; 3.8 \%)$. Surgical mortality was $0.8 \%(n=1)$.

Nineteen $(14.5 \%)$ were treated with radiotherapy. Twelve patients $(9.2 \%)$ were treated with medical therapy after surgery. Indications for medical therapy were adenoma persistence $(n=7)$ or progression $(n=1)$, visual disturbances $(n=5)$ and headaches $(n=3)$. The drugs used were bromocriptine $(n=4)$, cabergoline $(n=4)$ and octreotide $\operatorname{LAR}(n=3)$.

Immunohistochemical study for anterior pituitary hormones was performed in 123 tumor samples. In 47 patients $(38.2 \%)$ immunohistochemical staining was negative. Hormones most frequently detected were gonadotropins, followed ACTH and GH (Table 2).

Table 2. Clinical data of the patients at diagnosis and at last clinical visit.

\begin{tabular}{lll}
\hline & Basal & Last visit \\
\hline $\begin{array}{l}\text { Maximal tumor diameter (cm) } \\
(n=131)\end{array}$ & $2.6(2.0-3.1)$ & $0.9(0-1.8)^{*}$ \\
$\begin{array}{l}\text { Hypopituitarism } \\
(n=97)\end{array}$ & \\
$\begin{array}{l}\text { Total hypopituitarism (\%) } \\
\text { Partial (\%) }\end{array}$ & 8.2 & 9.3 \\
$\begin{array}{l}\text { Visual field examination } \\
(n=109)\end{array}$ & 46.4 & $53.6^{*}$ \\
Abnormal (\%) & & \\
& 53.2 & 19.3 \\
\hline
\end{tabular}

Note: Figures indicate the percentage of patients (\%) and/or the median (interquartile range). Willcoxon test for comparison of maximal tumor diameter and $\chi^{2}$ test for comparison of percentages of hypopituitarism and visual field examination presurgery and at last visit.

* Level of significance for multiple comparisons was adjusted using the Bonferroni correction to alpha: $p=0.0125$.

Immunohistochemical study for Ki-67 was performed in 54 patients $(\leq 2 \%$ in $38,3 \%$ in 11 and $\geq 5 \%$ in 5 patients) (Fig. 2). Tumors with Ki-67 index $\geq 3 \%$ were significantly associated with giant adenomas $\left(p=0.019 ; \chi^{2}\right.$ test $)$. 


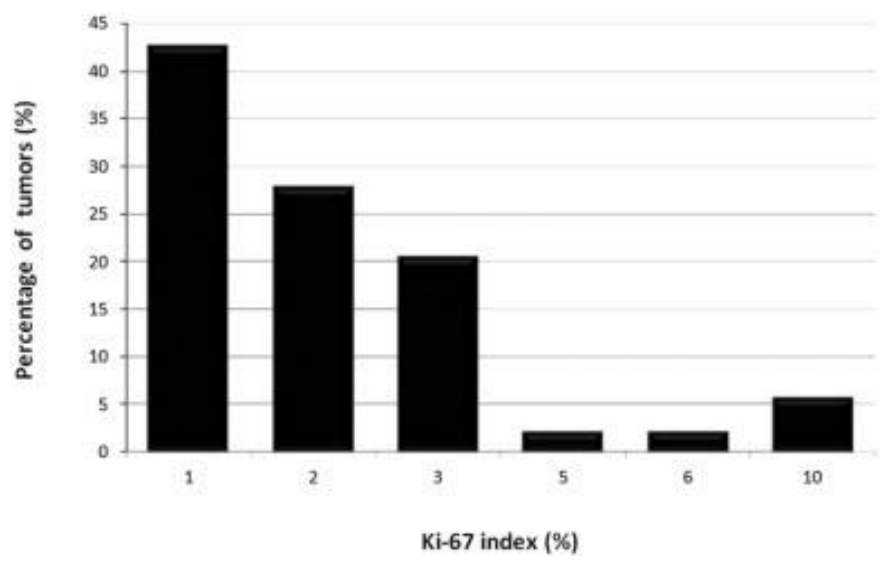

Fig. 2. Percent distribution of tumors according to Ki-67 index value $(n=54)$

\subsection{Clinical follow-up}

After a median follow-up of 57 months (25 to 128 months) maximal tumor diameter significantly decreased from $2.6 \mathrm{~cm}(2$ to $3.1 \mathrm{~cm})$ to $0.9 \mathrm{~cm}(0$ to $1.8 \mathrm{~cm}),(p<0.001$, Willcoxon test). At the end of follow-up, 20 out of 120 patients $(16.7 \%)$ had complete hypopituitarism and 49 patients (40.8\%) partial hypopituitarism. The most frequently affected hormonal axis was gonadal $(n=55 ; 45.8 \%)$, followed by thyroid $(n=50,41.7 \%)$ and adrenal $(n=42,35 \%)$ axes.

Visual field evaluation was abnormal in 24 out of 119 patients (20.2\%). The main visual field abnormality was unilateral temporal hemianopia $(n=6,5 \%)$, followed by bitemporal hemianopia $(n=5$, $5 \%)$ and bitemporal quadrantanopsia $(n=5,5 \%)$. Although those patients who presented alterations in the visual field showed a greater tumor size at diagnosis than those who did not, these differences were not statistically significant [3.0 cm $(2.1-3.5 \mathrm{~cm})$ vs $2.5 \mathrm{~cm}(2.0-3.0 \mathrm{~cm}) ; p=0.075]$.

Pituitary MRI in the last clinical visit was conducted in 112 patients and showed persistence of the tumor in most patients $(n=73,65.2 \%)$ and absence in the rest $(n=39,34.8 \%)$. There were no significant differences between these groups in relation to sex but there were in relation to age group at diagnosis $\left(p=0.03 ; \chi^{2}\right.$ test $)$. Young patients $(<40$ years $)$ had a prevalence of tumor persistence $\sim 6$ times higher than older patients ( $\geq 40$ years) $\left(p=0.03 ; \chi^{2}\right.$ test). Preoperative tumor size was similar in patients with and without tumor image in the last MRI [2.5 cm $(2.0-3.6 \mathrm{~cm})$ vs $2.7 \mathrm{~cm}(2.0-3.0 \mathrm{~cm})$; ns]. The percentage of patients with persistent tumor in the last MRI was $61.8 \%, 70.4 \%$ and $80 \%$ for those who underwent endoscopic endonasal surgery, microscopic transsphenoidal and transfrontal surgery, respectively.

At the end of the study, complete cure, defined as no tumor in the last pituitary MRI and normal pituitary function, was achieved in only 19 (17\%) of the 112 patients who could be evaluated. Those patients who met adenoma cure criteria showed a significantly lower preoperative tumor size than those that were not cured $[2.0 \mathrm{~cm}(1.6-2.7 \mathrm{~cm})$ vs $2.7 \mathrm{~cm}(2.1-3.6 \mathrm{~cm}) ; p=0.011$; Mann-Whitney test].

In the univariate Cox regression analysis only endoscopic endonasal surgery (HR 3.60, 95\% CI, 1.64$7.88, p=0.001)$ and radiotherapy (HR $0.09,0.01-0.67, p=0.019)$ behaved as positive and negative predictors, respectively, for the absence of tumor in the last MRI. In the multivariate analysis, both variables, endoscopic endonasal surgery (HR 2.74, 1.06-6.87, $p=0.036$ ) and radiotherapy (HR 0.04, $0.02-0.65, p=0.024)$ also behaved as positive and negative predictors, respectively, of tumor absence in the follow-up (Fig. 3). 


\section{Tumor absence}

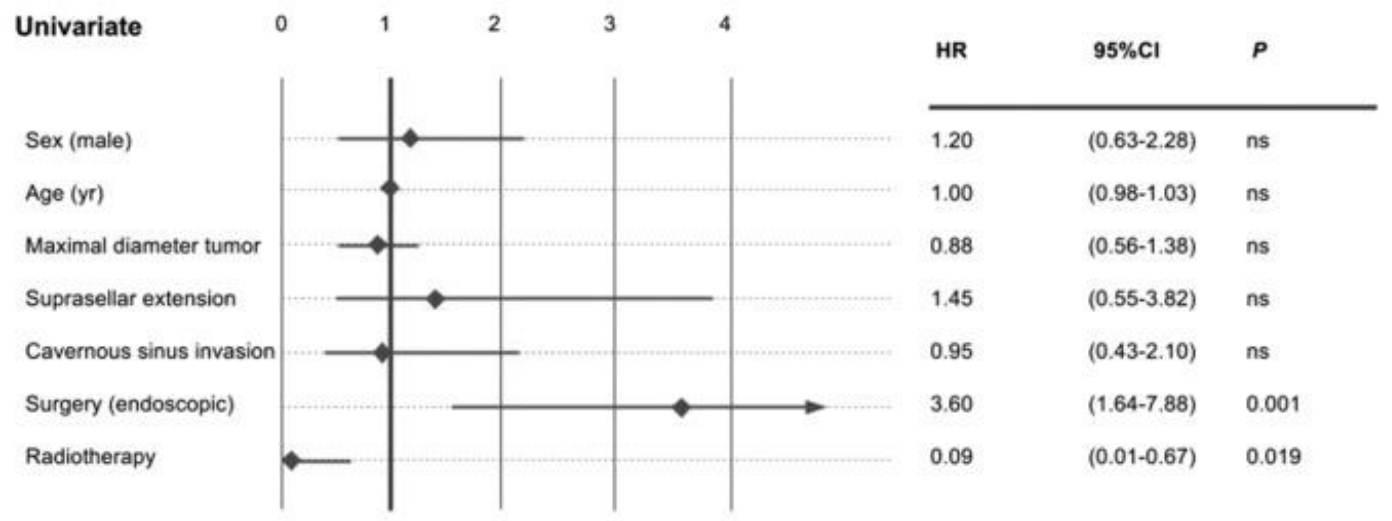

Multivariate

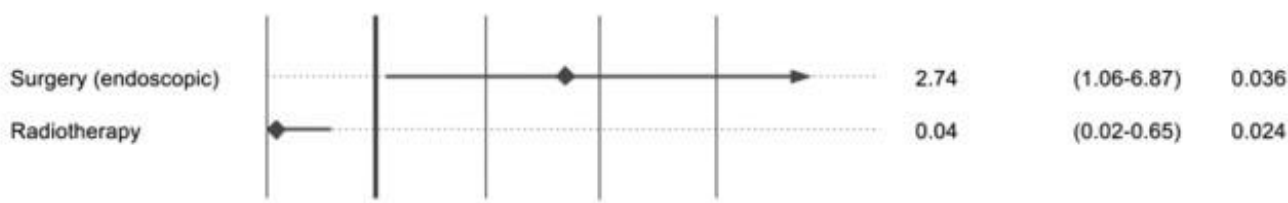

Fig. 3. Unadjusted and adjusted hazard ratios (HR with $95 \%$ confidence interval, CI) for tumor absence in the last clinical visit in NFPA patients underwent surgery classified according to several clinical variables.

In relation to complete cure rate, the univariate Cox regression analysis showed that endoscopic endonasal surgery (HR 6.84, 2.13-21.88, $p=0.001)$ and maximal diameter tumor (HR 0,45, 0.21-0.95, $p=0.035$ ) were positive and negative predictors, respectively. In the multivariate analysis, endoscopic endonasal surgery (HR 6.56, 1.76-24.5, $p=0.005$ ) behaved as the only positive predictor for complete cure in the follow-up, while maximal diameter tumor (HR 0.94, 0.89-0.99, $p=0.041$ ) was the only negative predictor (Fig. 4) 


\section{Complete cure}

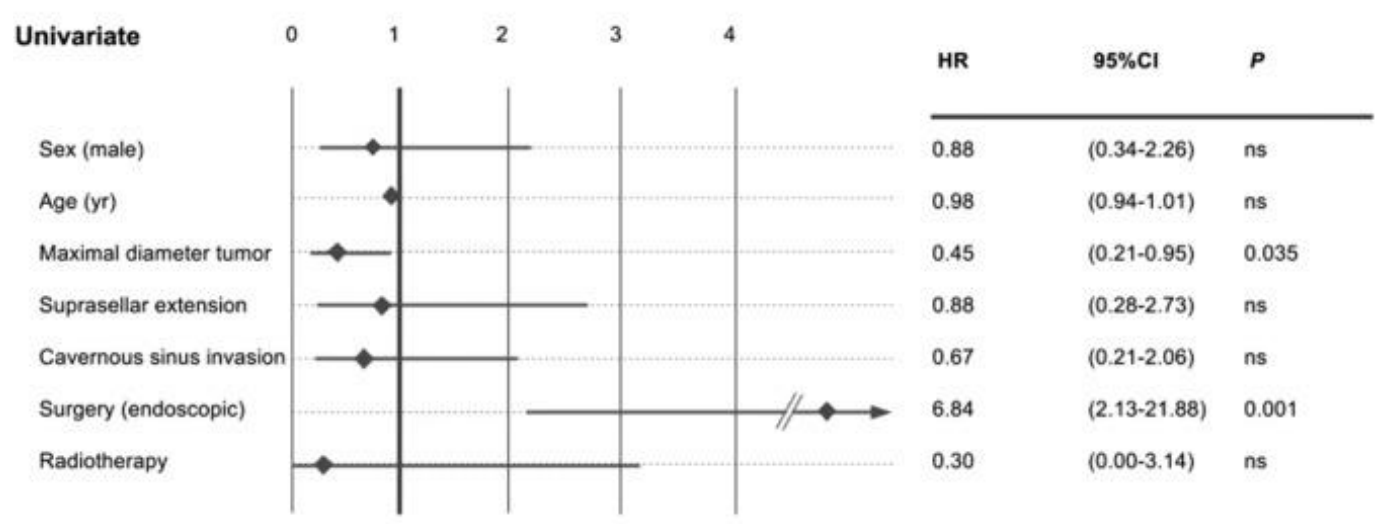

\section{Multivariate}

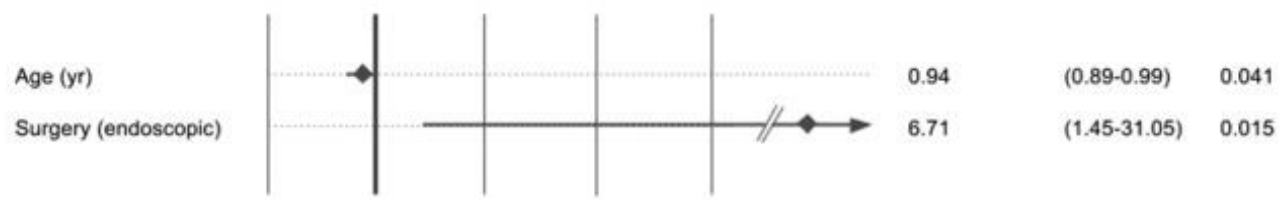

Fig. 4. Unadjusted and adjusted hazard ratios (HR with $95 \%$ confidence interval, CI) for complete cure in the last clinical visit in NFPA patients underwent surgery classified according to several clinical variables.

\section{Discussion}

The present study shows that surgical treated NFPAs mostly are pituitary macroadenomas starting symptomatically (headache and/or visual disturbances) in the sixth decade of life associated with hormone deficiencies in about half of patients. Although surgery controls tumor growth and improves visual disturbances, complete cure is unusual. Type of surgery is related to the disappearance of the adenoma and complete cure, being endoscopic endonasal surgery the main positive predictor for these clinical outcomes.

Our study suggests that symptomatic NFPAs are diagnosed between 50 and 55 years without sex predilection. These data differ slightly from other series from our country where age was slightly lower (fifth decade) and with a slight predilection for females, from 1.1 to $1.4(15,16)$. These differences might be explained by the different age in the analyzed cohorts. Some studies suggest that there is a sexual dimorphism in NFPAs in relation to the age at diagnosis [7]. It has been reported that before the fourth decade, NFPAs are more common in women, while from that age, males are predominant. Our population confirms this observation showing a slight predominance in females in patients < 40 years and in males in patients over 60 years; however, between 40 and 60 years, the prevalence was similar in both sexes (Fig. $1)$.

NFPA may occur in the context of a MEN1, although it is rare $(<5 \%)$ [18]. Our study shows that the prevalence of MEN1 in NFPAs underwent surgery is very low (1.5\%). 
The most common (50-60\%) form of clinical presentation of NFP macroadenomas are symptoms resulting from increased intracranial pressure, in the form of headaches and/or visual disturbances [11]. According to some recent surgical and non-surgical series, almost half of the NFPAs are incidental [19]. In our series these percentages were different ( $78 \%$ of the patients were symptomatic) probably due to the intrinsic characteristics of the cohort, since all our patients underwent surgery.

Although uncommon, endocrine symptoms recorded in our study were mainly related with gonadotropin deficiency, which is one of the most frequent hormone deficiency reported in these patients [15], [16], [19], [20]. The prevalence of hypopituitarism, partial or complete, at diagnosis was similar $(\sim 50 \%)$ to that described in other recently published series from a single hospital [19], [20].

The diagnosis of NFPA is performed by imaging tests, mainly MRI, which is the imaging test of choice [21]. In the surgical and nonsurgical series, the NFPA mean diameter is $\sim 2 \mathrm{~cm}$ [19]; slightly lower than found in our series (mean $2.8 \pm 1.1 \mathrm{~cm}$ ). This is probably due to the fact that in our study only patients who underwent surgery and, therefore with larger tumors, were evaluated. In our patients suprasellar extension of the adenoma was more frequent than the invasion of the cavernous sinus $(85 \%$ vs. $73 \%$ ), which implies a greater likelihood of compression of the optic pathways.

Both endoscopic endonasal and microscopic transsphenoidal approaches are recommended for symptom relief in patients with NFPAs [22]. Endoscopic endonasal surgery allows better visualization of areas of difficult access and larger tumor resections, and according some authors, it offers better surgical outcomes and lower complication rate [23], [24], [25], [26]. In our series remission rate ranged from 20\% for transfrontal surgery to $36 \%$ for endoscopic endonasal surgery. These differences could be explained at least partly by the inherent characteristics of adenoma (size and invasiveness) at the time of the diagnosis. In our series, endoscopic endonasal surgery was associated to a lower likelihood of tumor persistence during follow-up compared with other surgical techniques, and behaved as an independent positive predictor of long-term tumor absence and complete cure. These data, together with those reported in the literature, would support the use of endoscopic technique in the surgical management of NFPAs.

Postsurgical stereotactic radiotherapy achieves tumor disease stability in most (89-90\%) of the NFPAs; however, radiation therapy has been associated with a high percentage of new hormonal deficiencies (20-30\%) and, in some cases (1-13\%), new visual disturbances [11]. In our study, hypopituitarism was slightly higher, probably because we consider the different radiotherapy techniques (stereotactic and conventional) as a whole. Furthermore, combination therapy with surgery and radiotherapy was associated to a lower likelihood of tumor persistence in the follow-up compared to surgery alone.

The predictive value of Ki-67 index in NFPAs is controversial [11]. Some studies [27], [28], although not all [29], have found Ki-67 values more elevated in young patients ( $<30$ years) compared to older patients (> 40 years). Others have directly compared the rate of tumor growth with Ki-67 index in NFPAs [30], [31] finding an inverse correlation between $\mathrm{Ki}-67$ index and the doubling time of tumor size in recurrent NFPAs [30]. It has also been reported that a value of $\mathrm{Ki}-67 \geq 2 \%$ predicts an increased risk of progression of the residual adenoma after surgery, indicating the need to shorten the interval of neuroimaging study after surgery [32]. However, more recent studies have failed to show this association [33]. In our study, the value of Ki-67 was low $(\leq 2 \%)$ in the majority $(\sim 70 \%)$ of the patients and a high value $(\geq 3 \%)$ was not associated with age, sex, suprasellar extension or chiasm compression and the need for second surgery for tumor recurrence; however, it was associated with the presence of giant adenoma.

When we consider as cure criteria the absence of tumor and normal pituitary function, the percentage of cured patients was $<20 \%$. This is an interesting finding showing that a high percentage of patients persist with hormonal dysfunctions which implies a lifelong monitoring. As reviewed by Romijn et al., almost all patients who are appropriately treated for pituitary tumors enter a chronic phase with control or cure of hormonal excess, adequate treatment of pituitary insufficiency and relief of mass effects. This phase is associated with improvement of initial signs and symptoms, but also with the persistent 
consequences of the initial disease and associated treatments, leading to a chronic post-treatment syndrome [34].

Among the strengths of our study are the large number of patients evaluated and prolonged observation period analyzed. The main limitations are those derived from the type study, retrospective and multicenter, which involved the lack of uniformity in the therapeutic offer (different neurosurgeons and diverse surgical techniques) between hospital centers, and the absence of variables in some patients, such as Ki67.

In conclusion, this study shows that NFPA undergoing surgery is a sporadic and symptomatic pituitary macroadenoma associated to headache and visual disturbances, which is mainly diagnosed in the 4-6th decade of life, with no clear sex predilection. Hypopituitarism (partial or complete) at diagnosis occurs in about half of patients, being more frequent in males. Endoscopic endonasal surgery seems to behave as a positive predictor for the absence of tumor imaging and complete cure in the follow up. Adjuvant radiation therapy is occasionally used while medical treatment is used in exceptional cases. The cell proliferation index $\mathrm{Ki}-67$ is generally low $(<3 \%)$ and high value $(\geq 3 \%)$ is associated with cavernous sinus invasion and giant adenomas. Although surgery improves visual symptoms, pituitary function does not improve with time. Complete cure (absence of pituitary tumor and normal pituitary function) is uncommon so it requires a long-term monitoring.

\section{Conflict of interest}

The authors declare no conflict of interest and not received funding for this article.

\section{References}

[1] Ezzat S, Asa SL, Couldwell WT, Barr CE, Dodge WE, Vance ML, et al. The prevalence of pituitary adenomas: a systematic review. Cancer 2004;101:613 -9.

[2] Aflorei ED, Korbonits M. Epidemiology and etiopathogenesis of pituitary adenomas. J Neuro oncol 2014;117:379 -94.

[3] Dolecek TA, Propp JM, Stroup NE, Kruchko C. CBTRUS statistic al report: primary brain and central nervous system tumors diagnosed in the United States in 2005-2009. Neuro-Oncology 2012;14(Suppl. 5):v1-49.

[4] Shibui S. The present status and trend of brain tumors based on the data of the brain tumor registry of Japan. Brain Nerve 2012;64:28 6-90 [Shinkei Kenkyu no Shinpo ].

[5] Nilsson B, Gustavasson-Kadaka E, Bengtsson BA, Jonsson B. Pituitary adenomas in Sweden between 1958 and 1991: incidence, survival, and mortality. J Clin Endocrinol Metab 2000;85:14 20-5.

[6] Agustsson TT, Baldvinsdottir T, Jonasson JG, Olafsdottir E, Steinthorsdottir V , Sigurdsson G, et al. The epidemiology of pituitary adenomas in Iceland, 1955-2012: a nationwide populationbased study. Eur J Endocrinol 2015;173:655-64.

[7] Mindermann T, Wilson CB. Age-related and gender-related occurrence of pituitary adenomas. Clin Endocrinol (Oxf) 1994;41:359-64.

[8] Gruppetta M, Mercieca C, Vassallo J. Prevalence and incidence of pituitary adenomas: a population based study in Malta. Pituitary 2013;16:545-53.

[9] Saeger W, Ludecke DK, Buchfelder M, Fahlbusch R, Quabbe HJ, Petersenn S Pathohistological classification of pituitary tumors: 10 years of experience with the German pituitary tumor registry. Eur J Endocrinol 2007;156:203-16 [European Federation of Endocrine Societies ].

[10] Dekkers OM, Pereira AM, Romijn JA. Treatment and follow- up of clinically nonfunctioning pituitary macro adenomas . J Cl in Endocrinol Metab 2008;93:3717-26.

[11] Camara Gomez R. Non-functioning pituitary tumors: 2012 update. Endocrinol Nutr 2014;61:160-70.

[12] Greenman Y, Stern N. Optimal management of non-functioning pituitary adenomas. Endocrine 2015;50:51-5 
[13] Mayson SE, Snyder PJ. Silent pituitary adenomas. Endocrinol Metab Clin North Am 2015;44:79-87.

[14] Aghi MK, Chen CC, Fleseriu M, Newman SA, Lucas JW, Kuo JS, et al. Congress of neurological surgeons systematic review and evidence-based guidelines on the management of patients with non functioning pituitary adenomas: executive summary . Neurosurgery 2016;79:521-3.

[15] Marazuela M, Astigarraga B, Vicente A, Estrada J, Cuerda C, Garcia-Uria J, et al. Recovery of visual and endocrine function following transsphenoidal surgery of large non-functioning pituitary adenomas. J Endocrinol Invest 1994;17:703-7.

[16] Alameda C, Lucas T, Pineda E, Brito M, Uria JG, Magallon R, et al. Experience in management of 51 non-functioning pituitary adenomas: indications for post-operative radiotherapy. J Endocrinol Invest 2005;28:18-22.

[17] Civantos Modinoa S, Montaño Martínez J, López Serrano R, Díaz Guardiola P, Guijarro de Armasa G, Gómez Angulo JC, et al. Revisión casuística en el hospital universitario de Getafe de pacientes con patología hipofisaria adenomatosa intervenidos quirúrgicamente. Endocrinol Nutr 2010; 57: 306-10.

[18] Thakker RV, Newey PJ, Walls GV, Bilezikian J, Dralle H, Ebeling PR, et al. Clinic al practice guidelines for multiple endocrine neoplasia type 1 (MEN1). J Clin Endocrinol Metab $2012 ; 97$ : 2990-3011.

[19] Anagnostis P, Adamidou F, Polyzos SA, Efstathiadou Z, Panagiotou A, Kita M. Nonfunctioning pituitary adenoma s: a single center experience . Exp Clin Endocrinol Diabetes 2011;119:3 14-9.

[20] Fleseriu M, Bodach ME, Tumialan LM, Bonert V, Oyesiku NM, Patil CG, et al. Congress of neurological surgeons systematic review and evidence-based Guideline for pre-treatment endocrine evaluation of patients with nonfunctioning pituitary adenomas . Neuro surgery 2016; 79 :E52 7-9.

[21] Chen CC, Carter BS, Wang R, Patel KS, Hess C, Bodach ME, et al. Congress of neurological surgeons systematic review and evidence-based guideline on preoperative imaging assessment of patients with suspected nonfunctioning pituitary adenomas. Neurosurgery 2016;7 9:E5 24-6.

[22] Kuo JS, Barkhoudarian G, Farrell CJ, Bodach ME, Tumialan LM, Oyesiku NM, et al. Congress of neurological surgeons systematic review and evidence-based guideline on surgical techniques and technologies for the management of patient s with nonfunctioning pituitary adenomas. Neuro surgery 2016;79:E 536- 8 .

[23] Roelfsema F, Biermasz NR, Pereira AM. Clinical factors involved in the recurrence of pituitary adenoma s after surgical remission: a structured review and meta-analysis . Pituitary 2012;1 5:7 $1-83$.

[24] DeKlotz TR, Chia SH, Lu W, Makambi KH, Aulisi E, Deeb Z. Meta-analysis of endoscopic versus sublabial pituitary surgery. Laryngoscope $2012 ; 122: 511-8$.

[25] Gao Y, Zhong C, Wang Y, Xu S, Guo Y, Dai C, et al. Endoscopic versus microscopic transsphenoidal pituitary adenoma surgery: a meta-analysis. World J Surg Oncol 2014;12 (947819 -12-94).

[26] Jang JH , Kim KH , Lee YM, Kim J S, Kim YZ. Surgical results of pure endoscopic endonasal transsphenoidal surgery for 331 pituitary adenomas: an experience of a single institute for 15 years. World Neurosurg Dec 2016;96:545-55. http: //dx.doi.org/10.1016/j.wneu.2016.09.051.

[27] Yonezaw a K, Tamaki N, Kokunai T. Clinic al features and growth fractions of pituitary adenomas. Surg Neurol 1997; 48:494-500.

[28] Jaffrain-Rea ML, Di Stefano D, Minniti G, Esposito V, Bultrini A, Ferretti E, et al. A critical reappraisal of MIB-1 labelling index significance in a large series of pituitary tumours: secreting versus non-secreting adenomas. Endocr Relat Cancer 2002; 9: 103-13.

[29] Mastronardi L, Guiducci A, Puzzilli F, Maira G. Anterior pituitary adenomas in patients aged more than 65 years : analysis of growth fraction (using the MIB-1 monoclonal antibody) and of clinical features in comparison to younger patients. Clin Neurol Neurosurg 2002;10 4: 44-8.

[30] Ekramullah SM, Saitoh Y, Arita N, Ohnishi T, Hayakawa T. The correlation of Ki-6 7 staining indices with tumour doubling times in regrowing non- functioning pituitary adenomas. Acta Neurochir 1996; $138: 1449-55$.

[31] Honegger J, Prettin C, Feuerhake F, Petrick M, Schulte-Monting J, Reincke M. Expression of Ki-67 antigen in non functioning pituitary adenomas: correlation with growth velocity and invasive ness. J Neurosurg 2003;99:6 74-9.

[32] Matsuyama J. Ki-67 expression for predicting progression of postoperative residual pituitary adenomas: correlations with clinical variables. Neurol Med Chir 2012;52 : 563-9. 
[33] Sadeghipour A, Mahouzi L, Salem MM, Ebrahim- Nejad S, Asadi-Lari M, Radfar A, et al. Ki67 labeling correlated with invasion but not with recurrence. Appl Immunohistochem Mol Morphol Feb 9 2016. http://dx.doi.org/10.1097/PAI.0000000000000303.

[34] Romijn JA. The chronic syndromes after previous treatment of pituitary tumours. Nat Rev Endocrinol 2016; $12: 547-56$. 